\title{
Modulated antiproton fluxes for interstellar production models
}

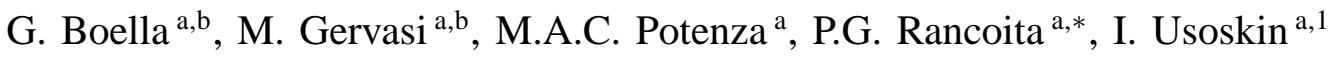 \\ a INFN-Milan, via Celoria 16, I-20133 Milan, Italy \\ ${ }^{\mathrm{b}}$ Department of Physics, University of Milan, I-20133 Milan, Italy
}

Received 12 January 1998; revised 26 March 1998; accepted 5 May 1998

\begin{abstract}
Expected differential fluxes of antiprotons at the Earth's orbit are calculated for minimum and medium strength of the solar modulation. We used an analytical solution in the force-field approximation. Local interstellar spectra are taken as predictions of different theoretical models of secondary production of antiprotons in the Galaxy. All the antiproton- as well as proton-fluxes are modulated in the same way for the purpose of a direct comparison in order to avoid additional uncertainties. It is shown that, in order to compare experimental data with the existing models predictions, next generation experiments should be able to measure fluxes of particles with energies above $100 \mathrm{MeV}$. (c) 1998 Elsevier Science B.V.
\end{abstract}

PACS: $96.40 . \mathrm{Kk}$

Keywords: Solar modulation; Antiproton spectrum

\section{Introduction}

Several balloon-borne experiments for detection of antiparticles (antiprotons) in cosmic rays were carried out since the late 1970 s (see, e.g., [1] and references therein). These observations have been performed by various groups and during different years (i.e. dissimilar phases of solar activity cycles). Therefore, it is difficult to compare different experimental results with each other.

There are a number of papers (e.g. [1-6]) where authors fit various theoretical Local Interstellar Spectra (LIS) of antiprotons to experimental data using different models of solar modulation. Since they use quite different modulation parameters (outer boundary, modulation strength, modulation model, etc.), un-

\footnotetext{
* Corresponding author. E-mail: Piergiorgio.Rancoita@mi.infn.it.

${ }^{1}$ Leave on absence from Ioffe Physical-Technical Inst., 194021 St. Petersbourg, Russian Federation.
}

certainties of expected modulated fluxes yielded by different modulation approaches might be even larger than differences of LIS fluxes calculated for different antiproton production models (Fig. 1). In order to compare expected fluxes at the Earth's orbit for different models of antiproton production in the Galaxy, it is necessary to calculate their modulation in the same way.

In the present paper, we have considered four different models of secondary production of antiprotons [2,3,6-8], as shown in Fig. 1. These models will be described in more details in Section 2. They differ for the choice of main parameters and their estimated values. In our study, calculations of the modulation were performed in the same way for all the four LIS', by using an analytical solution in the force-field approximation. This allows us to compare directly expected antiproton fluxes at the Earth's orbit and minimize possible uncertainties. For calculations of the Galac- 


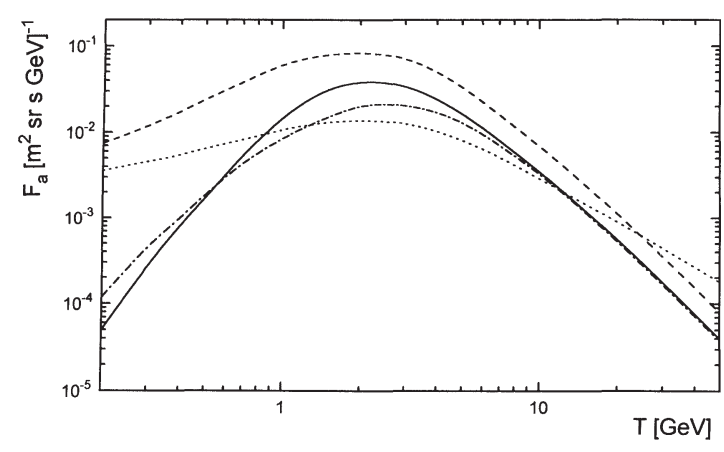

Fig. 1. Local Interstellar flux of antiprotons $\left(F_{a}\right)$ vs kinetic energy $(T)$, according to the models of secondary production: GS ( solid line), WP (dashed line), SH-DR (dotted line), SH-LB (dot-dashed line).

tic Cosmic Ray (GCR) proton flux at $1 \mathrm{AU}$ we used the proton LIS as given in [6] modulated in a similar way. Experimental spectra with small errors will be made available by the next generation of experiments from space. This way, the comparison between accurate modulated spectra and experimental data might untangle the relevant parameters and (hopefully) set their values.

We have calculated the modulation (Section 3 ) for two conditions: medium modulation ( the modulation strength parameter [9] $\Phi=750 \mathrm{MV}$ ) and minimum modulation (minimum of solar activity $\Phi=350 \mathrm{MV}$ ). For instance, the year 1998 is expected to be in the ascending phase of solar activity. In particular, the sunspot number is predicted to be as high as 70-100 in the middle of 1998 (NOAA data base; NASA GSFC; Australian Space Forecast Centre). However, since the modulation strength is delayed by several months with respect to solar activity, the conditions of 1998 are expected to be somewhat intermediate between our two calculations.

\section{Models of secondary antiproton production}

The detailed cosmic ray propagation model in the Galaxy, the galactic-halo diffusion model [10,11], has to be very complex in order to take into account observations.

Commonly used is the Leaky Box approximation (LBM) [12], for which the Galaxy is uniformly filled with energetic particles trapped for a long time. The particles' escape from the Galaxy is determined by diffusion (leakage). The standard steady state equilibrium equation for the Leaky box approximation $[13,14]$ can be written for antiprotons in the form as used by Protheroe [15],

$$
\begin{gathered}
Q(E)-\frac{N(E)}{\lambda_{\mathrm{esc}}(E)}-\frac{N(E)}{\lambda_{\text {int }}(E)}+\frac{K}{\langle m\rangle} \int \frac{d \sigma}{d E}\left(E, E^{\prime}\right) \\
\times N_{p}\left(E^{\prime}\right) d E^{\prime}-\frac{\partial}{\partial E}\left[\left\langle\frac{\partial E}{\partial x}\right\rangle N(E)\right]=0
\end{gathered}
$$

where $Q(E)$ is the source of primary antiprotons, second and third terms represent particles losses due to escape from the Galaxy and interactions with the interstellar medium, respectively. The integral term describes production of antiprotons by collisions of protons with the interstellar gas, factor $K$ (the nuclear enhancement factor) accounts for heavier nuclei. The last term of Eq. (1) represents energy losses for ionisation (see Ref. [14]) and inelastic collisions ( see [15] for details). The source term of antiprotons incoming from outside the Galaxy, $Q(E)$ in Eq. (1), is neglected.

The usual approach to solve Eq. (1) is to estimate the source term, to take into account nuclei heavier than proton, and to get an equilibrium spectrum. There are a number of papers where authors solved the Leaky Box approximation under different assumptions. These models differ for the choice of main parameters and their estimated values.

\subsection{LBM by Gaisser and Schaefer}

The model by Gaisser and Schaefer [2,3], thereafter GS, is the first LBM of secondary antiproton production in the Galaxy we have considered. Authors accurately reexamined most of the earlier assumptions of the secondary production models: the spectral index and normalization of interstellar primary protons, the nuclear enhancement factor as well as the time spent in the Galaxy by cosmic rays. They have also considered parameter uncertainties in the model. This way authors produced an accurate estimate of the GCR antiproton flux with the estimated model error bars.

The authors assumed a power law dependence of the escape time on particle rigidity, according to [16]. Furthermore, a leakage of antiprotons by nuclear interactions with the interstellar medium is considered. The authors neglected processes of particles reaccel- 
eration during the propagation in the Galaxy, according to Ref. [17]. They also neglected energy losses due to ionisation or scattering. The authors reconsidered the path length in the Galaxy. They adopted the following expression for the escape path length distribution [15], in order to take into account effects due to the path length decreasing at lower energies:

$\lambda_{e}=\Lambda\left[\frac{\beta}{\sqrt{4-3 \beta^{2}}}\right]^{n} P^{-\delta}$,

where $\beta$ and $P$ are the velocity (in the light speed units) and rigidity of particles, respectively, $\Lambda, n$ and $\delta$ are constants fitted to experimental data.

Gaisser and Schaefer estimated the sources of possible errors of the expected antiproton LIS. Since they used the worst combination of separate uncertainties, the final error bar of the model which is as high as factor 3 can be considered as an upper limit of the model uncertainties.

\subsection{LBM by Webber and Potgieter}

Another recent LBM comes from Webber an Potgieter [6] (WP thereafter). The main difference between WP and GS models is in the choice of proton LIS, path length distribution and inelastic crosssections. In particular, for the path length distribution the authors used the following expression (see [18]):

$$
\begin{array}{ll}
\lambda_{e}=10.83 \beta\left(P_{0} / P\right)^{-0.6}, & P>P_{0}, \\
\lambda_{e}=10.83 \beta & P<P_{0},
\end{array}
$$

where $P_{0}=4 \mathrm{GV}$. Webber and Potgieter also considered the effect of energy losses by inelastic scattering of antiprotons in the interstellar medium. The calculated flux of antiprotons is significantly higher than GS one. The authors did not estimate uncertainties of the model.

\subsection{Diffusive reacceleration model by Simon and Heinbach}

The effect of possible energy gain by transporting particles through the Galaxy was considered by Simon and Heinbach [7,8] (thereafter SH-DR). The authors suggested a process of diffusive reacceleration of GCR by means of the second order Fermi mechanism [19], occurring in the interstellar turbulent plasma, responsible for the diffusion of cosmic rays in the Galaxy [20]. A Kolmogorov spectrum has been assumed for the magnetic field inhomogeneities.

The authors fit experimental data by a Leaky Box model with reacceleration added. The diffusive reacceleration process modifies the GCR spectra, especially in the lower energy region. In fact, for low energy particles the process of energy gain is effective while for more energetic particles the diffusive propagation dominates. This effect produces an interstellar flux of antiprotons larger by at least one order of magnitude at $100 \mathrm{MeV}$ than the spectrum predicted without the reacceleration process (see, for comparison, the spectrum considered in Subsection 2.4). Furthermore, the predicted spectral maximum is flattened and shifted at lower energies (Fig. 1).

\subsection{LBM by Simon and Heinbach}

The last model considered, in the present paper, is a simple Leaky Box model realized by Simon and Heinbach [7] (SH-LB). This model makes use of more recent data than the ones utilized in the classical Protheroe [15] Leaky Box model. However, it uses a few more simplified approaches than the previous models (GS and WP). In particular, the authors choose the nuclear enhancement factor and other model parameters without a detailed discussion. On the other hand, this model, being the same used to build the SH-DR model, is particularly suitable to be compared with it.

\section{Solar modulation of antiproton spectra}

In order to compare fluxes of antiprotons (predicted by production models) near the Earth, we need to account the solar modulation of GCR flux during the propagation through the heliosphere.

\subsection{Calculation techniques}

The transport of GCR in the heliosphere is described with the transport equation [21] which can be written for a spherically-symmetric steady state case as 
$\frac{1}{r^{2}} \frac{\partial}{\partial r}\left(r^{2} \kappa \frac{\partial f}{\partial r}\right)-V \frac{\partial f}{\partial r}+\frac{1}{r^{2}} \frac{\partial}{\partial r}\left(r^{2} V\right) \frac{P}{3} \frac{\partial f}{\partial P}=0$,

where $f$ is the omnidirectional distribution function of particles (number of particles per unit volume of phase space $\left(d^{3} r d^{3} p\right)$ averaged over particle directions), $P$ is rigidity, $r$ is position, $V$ represents the radially directed solar wind velocity (usually $\boldsymbol{V}$ is assumed to be $400 \mathrm{~km} \mathrm{~s}^{-1}$ in the ecliptic plane), and $\kappa$ is the diffusion coefficient.

As an approximation of Eq. (4), the so-called forcefield approximation $[9,22]$ is usually considered,

$\frac{\partial f}{\partial r}+\frac{V P}{3 \kappa} \frac{\partial f}{\partial P}=0$.

The validity of this approximation was proven in [23]. It is known (e.g. [24]), that the solution of such equation can be found in the form of characteristics curves which are lines of constant $f$ in the $(r, P)$ plane. The characteristics curves are given by the expression

$\frac{d P}{d r}=\frac{V P}{3 \kappa}$.

Following the standard quasilinear theory (see for instance [4]), we can consider $\kappa=A \cdot \beta P$, where $\beta$ is the particle velocity in the units of the light speed. In this case, the solution of Eq. (6) is

$T_{R}-T(r)=\Phi \cdot \frac{R-r}{R-1}$,

where $T_{R}$ and $T(r)$ is the kinetic energy of a particle at the distance $R$ and $r$, respectively; $R$ is the outer heliospheric boundary (in AU), $\Phi=V(R-1) / 3 A$ is the strength of modulation. For $r=1 \mathrm{AU}$, one can easily obtain that $f_{1 \mathrm{AU}}\left(T_{R}-\Phi\right)=f_{R}\left(T_{R}\right)$. Thus, the differential particle intensity, $j=f \cdot P^{2}$ at $1 \mathrm{AU}$ is given like

$j_{1 \mathrm{AU}}\left(T_{R}-\Phi\right)=j_{R}\left(T_{R}\right) \frac{\left(T_{R}-\Phi\right)\left(T_{R}-\Phi+2 T_{o}\right)}{T_{R}\left(T_{R}+2 T_{o}\right)}$,

where $T_{o}=932 \mathrm{MeV}$ is the rest energy of a proton.

Following the observations of the mean free path of solar particles, it is also considered that the diffusion coefficient becomes rigidity independent for lower energies,
$\kappa=A \cdot \beta P, \quad$ for $P>P_{c}$,

$\kappa=A \cdot \beta P_{c}, \quad$ for $P<P_{c}$.

where $P_{c}$ is $\approx 1 \mathrm{GV}$ (see, e.g., Ref. [4]).

In this case Eq. (6) for the characteristics curve, $f=$ const., becomes of the form

$\frac{d P}{E}=\frac{V}{3 A P_{c}} d r$,

where $E$ is the total particle energy. The solution of Eq. (9), for $r=1 \mathrm{AU}$, is

$E_{R}-E_{c}=P_{c} \ln \left(\frac{P_{1 \mathrm{AU}}+E_{1 \mathrm{AU}}}{P_{c}+E_{c}}\right)+\Phi$,

where rigidity is expressed in $\mathrm{MV}$, and energy in $\mathrm{MeV}$, respectively (see also [4]). Thus, the expression for $P_{1 \text { AU }}$ can be obtained from Eq. (10) as

$P_{1 \mathrm{AU}}=\frac{1}{2}\left(\phi-\frac{T_{o}^{2}}{\phi}\right)$,

where $\phi\left(T_{R}, P_{c}, \Phi\right)=\left(P_{c}+E_{c}\right) \exp \left[\left(T_{R}-T_{c}-\right.\right.$ $\left.\Phi) / P_{c}\right]$.

Note that the force-field approximation (Eq. (5) and correspondingly Eqs. (8) and (10)) works well for the energy range above a few hundred $\mathrm{MeV}$ and should not be used for particles with energy less than $100 \mathrm{MeV}$. The difference between Eqs. (8) and (10), standard quasilinear theory and observed solar cosmic rays mean free path, respectively, becomes remarkable at the energy range below several hundred MeV's.

\subsection{Results of calculations and comparison to other modulation estimates}

For calculating the modulation effect, we have used the following parameter values: the solar wind velocity $V=400 \mathrm{~km} / \mathrm{s}$, the outer border of the heliosphere $R=100 \mathrm{AU}$, the modulation strength $\Phi=350 \mathrm{MV}$ ( minimum modulation) and $\Phi=750 \mathrm{MV}$ (medium modulation).

The modulated fluxes of galactic antiprotons for four different antiproton secondary production models (GS, SH-DR, SH-LB, WP) at the Earth's orbit are shown in Fig. 2. In Fig. 2a modulation is evaluated for minimum activity ( $\Phi=350 \mathrm{MV}$ ) while in Fig. $2 \mathrm{~b}$ for medium $(\Phi=750 \mathrm{MV})$ modulation strength. 

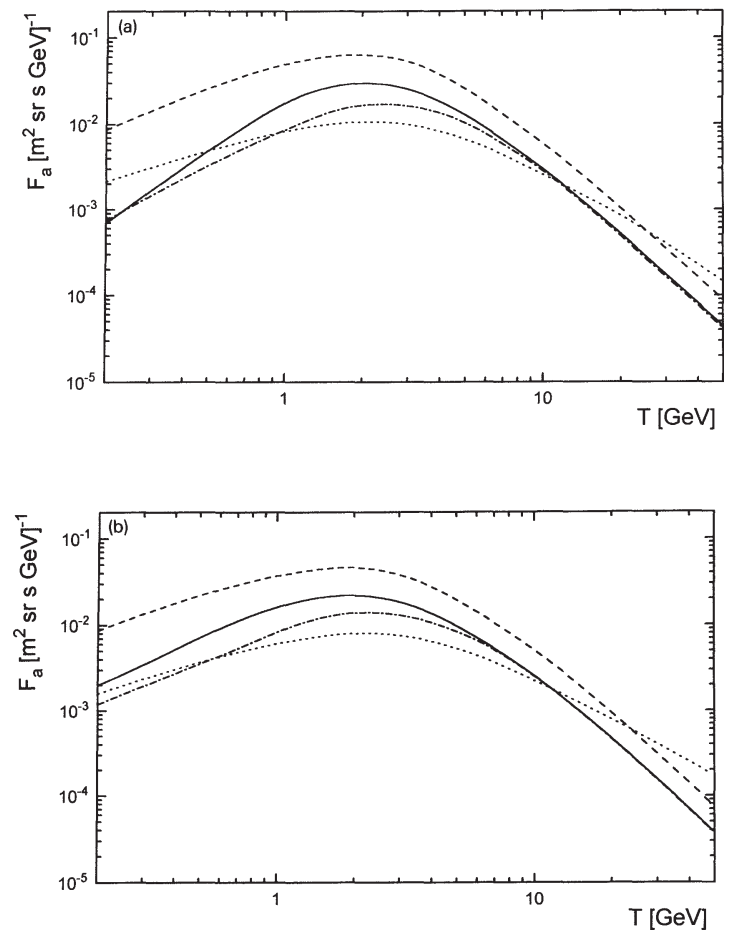

Fig. 2. Expected flux of antiprotons $\left(F_{a}\right)$ vs kinetic energy $(T)$ at the Earth's orbit for the four Galactic antiproton production models: GS ( solid line), WP (dashed line), SH-DR (dotted line), SH-LB (dot-dashed line). Weak and medium modulation conditions (see text) are considered in Fig. 2a and in Fig. 2b, respectively.

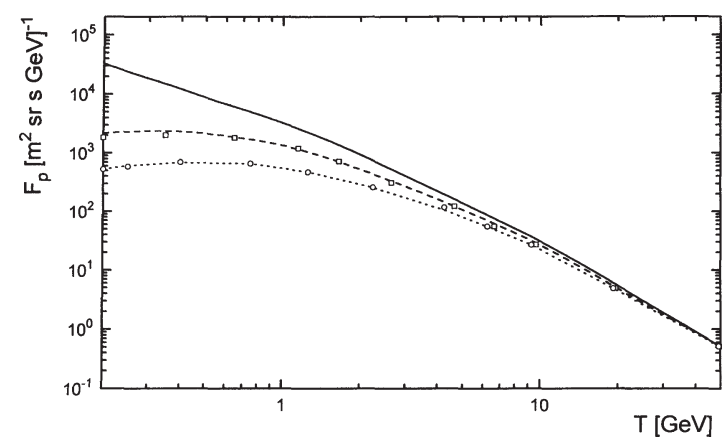

Fig. 3. Local Interstellar flux of protons $[1,6], F_{p}$ (solid line), together with expected modulated flux at the Earth's orbit are shown. Our results for weak (dashed line) and medium (dotted line) modulation conditions are compared with the results by Labrador and Mewaldt [1] (squares and circles, respectively) for the same conditions.
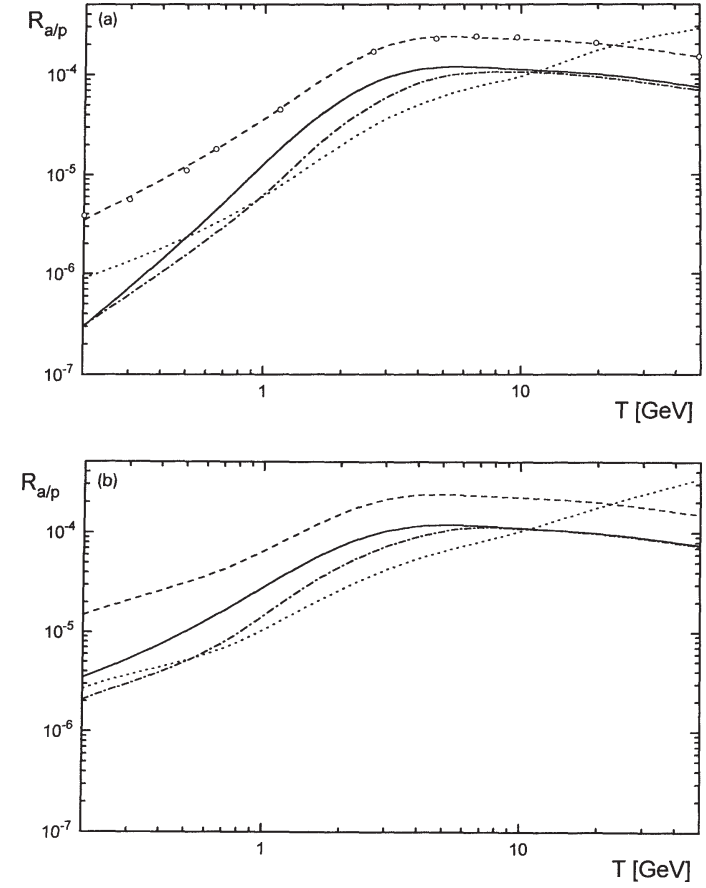

Fig. 4. Expected antiproton-to-proton ratio $\left(R_{a / p}\right)$ at the Earth's orbit for the four models of secondary antiproton production: GS ( solid line), WP (dashed line), SH-DR (dotted line), SH-LB (dot-dashed line). Weak and medium modulation conditions are considered in Fig. 4a and in Fig. 4b, respectively. For the weak modulation conditions our result for WP model is compared to the ratio calculated in [1] for the same conditions (circles).

We have adopted the proton LIS according to Ref. [1,6] to estimate the proton flux at the Earth's orbit. The modulated fluxes of protons for the two modulation strengths are presented in Fig. 3. We can evaluate, also, antiproton to proton ratios for different modulation conditions. These ratios at the Earth's orbit are shown in Fig. $4 \mathrm{a}$ - for the minimum, and in Fig. $4 \mathrm{~b}$ - for the medium modulation.

Results obtained by our modulation technique have been compared with spectra obtained recently by Labrador and Mewaldt [1] for the same modulation strength. These authors solved numerically Eq. (4) in order to evaluate the effect of modulation on galactic particles.

In Fig. 3, our results are shown together with the modulated proton fluxes near the Earth obtained in paper [1]. The curves are in good agreement for the energy range above $500 \mathrm{MeV}$. A slight difference (within 20\%) for the weak modulation conditions at 
lower energies appears as a result of the force field approximation used in our calculations. For the medium modulation conditions both curves agree within $10 \%$ for the entire energy range. We conclude that our results, obtained by the analytical solution of the force field approximation, are in good agreement with earlier published modulated proton spectra obtained with a numerical solution of the transport equation.

The curves (indicated by WP in Figs. 2a and 2b) represent antiproton spectra near the Earth, once modulated following our procedure, for the LIS model by Webber and Potgieter [6]. These modulated spectra are in good agreement with fluxes obtained by Labrador and Mewaldt [1] under similar modulation conditions. In fact, we have compared the expected antiproton-to-proton ratio, $R_{a / p}$, obtained in our calculations and in [1] for the same conditions (see Fig. 4a).

In summary, our results for both protons and antiprotons (WP model) agree with earlier published spectra obtained under similar conditions. Calculated fluxes of antiprotons for other models differ from earlier published results owing to different modulation conditions.

\subsection{Modulation uncertainties}

Even if the calculation itself is accurate, the knowledge of the modulation effect, in particular on antiprotons, is approximate. Uncertainties of several tens of percent are expected for the modulated flux of particles with kinetic energy of (100-200) MeV, becoming smaller for higher energies.

Below a few GeV's of kinetic energy, there are large proton LIS uncertainties (about an order of magnitude at $200 \mathrm{MeV}$ ). However, this is not crucial for the modulated proton spectrum owing to the adiabatic energy loss effect. In fact, uncertainties of the proton spectrum at the Earth orbit have been estimated (see Ref. [1]) to be less than $10 \%$ for the investigated energy range.

Furthermore, when the force-field approximation is exploited, an additional source of uncertainties comes from effects related to charge drift (e.g., see $[6,25,26])$. These effects result in a different modulation for protons and antiprotons, in particular during periods of minimum solar activity. A model estimate (Ref. [27]) indicates uncertainties on the antiproton to proton ratio (due to the charge drift effect) to be less than $20 \%$ above $200 \mathrm{MeV}$. They decrease with increasing energy (being less than $10 \%$ for energies above $500 \mathrm{MeV}$ ). However, for medium modulation conditions when the disturbances of heliospheric neutral sheet are larger, the sign-charge effect becomes smaller and the resulting uncertainties are reduced to about $10 \%$ for particle energies above $200 \mathrm{MeV}$ (e.g., see [26]). This effect is negligible under strong solar modulation. It has to be noted that since the charge particle drift effect reduces the particle flux, the antiproton to proton ratio will vary, to a first approximation, by a similar percentual amount. However, it can be increased or decreased depending on the solar cycle.

A further detailed discussion on both modulation uncertainties and relevance of the charge drift effect can be found in Ref. [1].

\section{Discussion}

As discussed above, uncertainties of LIS models are large (see Section 2.1 and Ref. [2] ). In addition, these ones have to be combined with uncertainties introduced by the modulation procedure (as pointed out in Section 3.3). However, when a large statistics set of experimental data allows the determination of a precise antiproton energy distribution, an indication of the preferred LIS model can be obtained by fitting predicted modulated spectra to experimental data.

The Local Interstellar Spectrum of antiprotons, according to Webber and Potgieter model [6], is much higher than others - the difference being as high as two orders of magnitude in the lower energy range (Fig. 1). The behaviour of lower energy part of the particle spectra at $1 \mathrm{AU}$ is mainly related to the middle energy part of LIS. In fact, the modulation includes the effect of "pumping" particles into lower energy range because of the process of adiabatic energy losses. Large difference between WP and other model spectra becomes less significant once modulated. One can see that the maximum difference between these modulated spectra is about one order of magnitude at minimum modulation (Fig. 2a), but it becomes even less (by factor 6) at medium modulation (Fig. 2b).

The raise of the energy distribution could be related to a possible diffusive reacceleration, as it can be seen 
in the low energy part of the curve SH-DR in Fig. 2a. Thus, as mentioned above, accurate experiments on detection of antiprotons near the Earth should be performed to study different production models.

At medium solar activity, the modulated WP spectrum is still much higher than all the other ones, even when modulation uncertainties are taken into account. Consequently, it can be untangled from the other spectra when a comparison with the next generation experimental data is carried out. However, under such a modulation condition, all the other LIS spectra, because they are quite close to each other (Fig. 2b), can be hardly untangled by comparing to experimental data.

A similar situation occurs with regard to calculated ratios of antiproton-to-proton fluxes at the Earth's orbit (Fig. 4). The ratio for WP differs largely from the other ones, irrespective to the exact period of solar activity. However, minimal modulation seems to be needed in order to compare model predictions with experimental data for energies up to several GeV's.

While the dependence of antiproton flux on the modulation strength is relatively weak (compare Figs. 2a and 2b), the proton flux (Fig. 3) is heavily dependent on it. In fact, because of the shape of LIS' and the effect of "pumping" particles into lower energy range, the proton flux near the Earth varies (under different modulation conditions) by a larger amount than what happens to antiprotons. This leads to a large variation of the expected ratio, $R_{a / p}$, from minimum to medium modulation conditions, and therefore to larger uncertainties with respect to the antiproton fluxes. On the other hand, in the past experimental data were presented in the form of spectral ratio, $R_{a / p}$.

In general, differences between fluxes of antiproton predicted by different models of secondary antiproton production are larger in the lower energy range (below $1 \mathrm{GeV}$ ). Thus, a dedicated experiment with large sensitivity might be able to untangle the most suited LIS production model. Furthermore, the measurement of the flux of antiprotons, with energies larger than $1 \mathrm{GeV}$, allows us to investigate details of solar modulation mechanisms with respect to antiprotons.

\section{Acknowledgements}

We thank Dr. G.A. Kovaltsov for useful comments and discussion. We would like to thank also Prof. C. Paizis and Dr. G. Bonelli for suggestions and proposals for improvements.

\section{References}

[1] A.W. Labrador, R.A. Mewaldt, Astrophys. J. 480 (1997) 371.

[2] T.K. Gaisser, R.K. Schaefer, Astrophys. J. 394 (1992) 174.

[3] T.K. Gaisser, R.K. Schaefer, Adv. Space Res. 19 (1997) 775.

[4] J.S. Perko, Astron. Astrophys. 184 (1987) 119.

[5] J.S. Perko, Astrophys. J. 397 (1992) 153.

[6] W.R. Webber, M.S. Potgieter, Astrophys. J. 344 (1989) 779.

[7] M. Simon, U. Heinbach, Astrophys. J. 456 (1996) 519.

[8] U. Heinbach, M. Simon, Astrophys. J. 441 (1995) 209.

[9] L.J. Gleeson, W.I. Axford, Astrophys. J. 154 (1968) 1011.

[10] V.L. Ginzburg, V.S. Berezinskii, S.V. Bulanov, V.A. Dogiel, V.S. Ptuskin, Astrophysics of Cosmic Rays (Elsevier, Amsterdam, 1990).

[11] I. Lerche, R. Schlickeiser, Astrophys. J. 239 (1980) 1089.

[12] T.K. Gaisser, Cosmic Rays and Particle Physics (Cambridge Univ. Press, Cambridge, 1990).

[13] V.L. Ginzburg, S.I. Syrovatskii, The Origin of Cosmic Rays (Pergamon, Oxford, 1964)

[14] L.C. Tan, L.K. Ng, J. Phys. G 9 (1983) 227.

[15] R.J. Protheroe, Astrophys. J. 251 (1981) 387.

[16] J.F. Ormes, R.J. Protheroe, Astrophys. J. 272 (1983) 756.

[17] M. Simon, U. Heinbach, C. Koch, Astrophys. J. 320 (1987) 699.

[18] M. Gupta, W.R. Webber, Astrophys. J. 340 (1989) 1124.

[19] E. Fermi, Phys. Rev. 75 (1949) 1169.

[20] M.A. Forman, J.R. Jokipii, A.J. Owens, Astrophys. J. 208 (1974) 535.

[21] E.N. Parker, Planet. Space Sci. 13 (1965) 9.

[22] L.A. Fisk, J. Geophys. Res. 76 (1971) 221.

[23] L.A. Fisk, W.I. Axford, J. Geophys. Res. 74 (1969) 4973.

[24] E. Kamke, Differentialgleichungen, Losungsmethoden und Losungen (Leipzig, 1959).

[25] J. Kota, J.R. Jokipii, Astrophys. J. 265 (1983) 573.

[26] R.A. Burger, M.S. Potgieter, Astrophys. J. 339 (1989) 501.

[27] J.P.L. Reinecke, M.S. Potgieter, J. Geophys. Res. 99 (1994) 14761. 